\title{
Effectiveness of a web-based health risk assessment with individually-tailored feedback on lifestyle behaviour: study protocol
}

\author{
Eva K Laan ${ }^{1,2^{*}}$, Roderik A Kraaijenhagen ${ }^{2}$, Niels Peek ${ }^{3}$, Wim B Busschers ${ }^{1}$, Marije Deutekom ${ }^{1}$, Patrick M Bossuyt ${ }^{4}$,
} Karien Stronks ${ }^{1}$ and Marie-Louise Essink-Bot ${ }^{1}$

\begin{abstract}
Background: Physical inactivity, unhealthy dietary habits, smoking and high alcohol consumption are recognized risk factors for cardiovascular disease and cancer. Web-based health risk assessments with tailored feedback seem promising in promoting a healthy lifestyle. This study evaluates the effectiveness of a web-based health risk assessment with individually-tailored feedback on lifestyle behaviour, conducted in a worksite setting.
\end{abstract}

Methods/Design: The web-based health risk assessment starts with a questionnaire covering socio-demographic variables, family and personal medical history, lifestyle behaviour and psychological variables. Prognostic models are used to estimate individual cardiovascular risks. In case of high risk further biometric and laboratory evaluation is advised. All participants receive individually-tailored feedback on their responses to the health risk assessment questionnaire. The study uses a quasi-experimental design with a waiting list control group. Data are collected at baseline (T0) and after six months (T1). Within each company, clusters of employees are allocated to either the intervention or the control group. Primary outcome is lifestyle behaviour, expressed as the sum of five indicators namely physical activity, nutrition, smoking behaviour, alcohol consumption, and symptoms of burnout. Multilevel regression analysis will be used to answer the main research question and to correct for clustering effects. Baseline differences between the intervention and control group in the distribution of characteristics with a potential effect on lifestyle change will be taken into account in further analyses using propensity scores.

Discussion: This study will increase insight into the effectiveness of health risk assessments with tailored feedback and into conditions that may modify the effectiveness. This information can be used to design effective interventions for lifestyle behaviour change among employees.

Trial registration: Dutch Trial Register NTR8148.

Keywords: Ehealth, Lifestyle behaviour, Health risk assessment, Worksite health promotion

\section{Background}

Most people in western European populations do not meet current recommendations for healthy behaviour, in terms of sufficient physical activity, healthy dietary habits, limited alcohol consumption and non-smoking [1]. For example, in $2008,37 \%$ of European adults aged $\geq 15$ years was insufficiently active, i.e. less then $150 \mathrm{~min}$ of moderate physical activity per week and the prevalence

\footnotetext{
* Correspondence: e.k.laan@amc.uva.nl

'Department of Public Health, Academic Medical Center, Amsterdam, The Netherlands

Full list of author information is available at the end of the article
}

of daily tobacco smoking among this population was nearly $29 \%$.

In the Netherlands, in 2007, according to the Dutch Healthy Physical Activity Guideline (30 min/day, 5 days/ week at moderate intensity level) $41 \%$ of the population aged $\geq 18$ years was insufficiently active, and $84 \%$ was insufficiently active according to the cardio-respiratory fitness guideline of $20 \mathrm{~min} /$ day, 3 days/week at high intensity level $[2,3]$. Results of the Dutch national food consumption survey in 2003 showed that, among young adults (1930 years), only $5.5 \%$ of men and $0.2 \%$ of women consumed the recommended amount of vegetables (150 g/day) and 
only $7-8 \%$ consumed the recommended amount of fruit (200 g/day) [4]. Smoking and drinking are also still common behaviours. In 2008, 27\% of the population aged $\geq 15$ years was smoking, and $14 \%$ of men and $11 \%$ of women aged $\geq 12$ years were drinking too much alcohol [5].

Unhealthy behaviour is known to be a risk factor for chronic conditions such as diabetes, cardiovascular diseases and cancer [5]. Therefore, promoting healthy behaviour has become a central goal of public health policy. Various programs have been developed to promote healthy behaviour and lifestyle change. Offering health promotion interventions via the worksite allows reaching large groups of adults, because most spend a large portion of their waking hours at their workplace [6]. Health promotion via the worksite has the additional advantages of enabling the introduction of social support, and making use of a natural social network for peer support [7]. Health promotion via the worksite may also offer opportunities to make environmental and structural changes that support healthy behaviour [8]. A healthier lifestyle, with the prevention of related (chronic) diseases, not only benefits employees and society, but also employers, in the form of less sickness absence and lower productivity losses [7].

The health risk assessment (HRA) is a commonly used instrument in the promotion of healthy behaviour [9]. A recent review showed that a HRA is useful as a gateway intervention to broader worksite health promotion programs [9]. HRAs screen for risk factors of disease and provide feedback on those risks. Many HRAs now make use of the Internet, which allows delivering individuallytailored interventions [10]. For example, within the worksite setting, emails and web pages can reach broad and diverse employee populations [11]. With web-based HRAs, employees are also able to apply the program in privacy, e.g. at home, and at a time that suits them [12]. However, although HRAs are promising, there is limited knowledge about what actually makes them work [8].

The primary aim of this study is to evaluate the effectiveness of a web-based HRA with individually-tailored feedback conducted in a worksite setting, on lifestyle behaviour. A secondary aim is to explore conditions that could modify the effectiveness in specific settings or subgroups. The results of this study will contribute to the evidence base for web-based HRAs with tailored feedback aimed at changing health behaviour.

\section{Methods/Design Study design}

A quasi-experimental study will be conducted with a waiting list control group, carried out in a worksite setting. The study population consists of employees of companies that embedded the HRA in their corporate health management strategy. Data are collected at baseline (T0) and after six months (T1). Data collection started in
April 2011. According to Dutch law, medical ethical approval of the study was not required, as confirmed by the Medical Ethics Committee of the Academic Medical Centre of the University of Amsterdam. All necessary precautions to protect privacy of subjects were taken.

\section{Intervention}

The intervention is called the 'Prevention Compass'. It has been developed by the NDDO Institute for Prevention and Early Diagnostics (NIPED) in Amsterdam. The Prevention Compass consists of a web-based HRA with tailored feedback. Participation in the intervention is on a voluntary basis. All current employees of a participating company are invited to participate in the intervention. Excluded are pregnant women or women with a pregnancy in the past 6 months because, in this group, such measurements could result in abnormal values.

\section{Invitation for the intervention}

Employees are invited through a letter sent to their home address. An information leaflet with a description of the intervention is enclosed. The invitation letter informs employees that participation is voluntary, involves no personal costs, and that all personal data are treated confidentially, meaning that no data are shared with their employer, and that the data are used in an anonymous form for the present study only. No written informed consent is obtained, due to the fact that the intervention is online. Completion of the questionnaire is regarded as informed consent to participation. The invitation letter contains an activation code for registration at the intervention's website http://www.preventiekompas.nl. For each participant, a personal online health portal is made available. Here participants can fill in the questionnaire, order a box with measurement tools (see below), and view their results of the HRA. After registration, participants receive a personal number for secure access to their portal. In case of no response to the letter of invitation, invitees receive a single reminder after three weeks, in the form of a second letter to their home address.

\section{Web-based health risk assessment}

The web-based HRA starts with a questionnaire, covering socio-demographic variables, family and personal medical history, lifestyle behaviour (physical activity, smoking behaviour, alcohol consumption, dietary pattern) and psychological variables (stress, burnout, depression). Individual scores are used to estimate cardiovascular disease risk using validated risk scores. All risk calculations are based on prevailing practice guidelines, including the European and Dutch guidelines for cardiovascular risk management $[13,14]$. Individuals at increased risk for cardiovascular disease, diabetes and kidney disease are advised to perform additional biometric and laboratory evaluation. For this, participants can order a box with measurement tools via their portal. This box includes a 
sphygmometer, a measuring tape and instruction cards for measurement of blood pressure and waist circumference. Participants also receive a lab box with materials to collect a urine sample for cardio-metabolic measurements. All participants who ordered the lab box visit a central facility where urine samples can be handed in, and a blood sample is taken for analysis of total cholesterol, LDL-cholesterol, HDL-cholesterol, triglycerides, glucose, creatinin, urinary albumin to creatinin ratio, and HbA1C. Results are electronically transferred to the central HRA database. After additional measurements, results are used to recalculate the cardio-metabolic risk, define kidney function using the MRDR formula, i.e. to estimate the glomerular filtration rate (eGFR) and albumin-creatinin ratio and detect (pre)diabetes based on glucose and $\mathrm{HbA} 1 \mathrm{C}$ values.

For system security and data protection reasons, personal identification data and HRA data are stored on separate servers. An electronic firewall is placed between the servers and the Internet. Only participants certified by ID and password are able to access the servers.

\section{Tailored feedback}

\section{Theoretical framework for lifestyle change}

The tailored feedback provided as part of the intervention is based on Prochaska's transtheoretical model [15]. According to this model, behavioural change is a process consisting of five subsequent phases: precontemplation (no intention of behaviour change between one and six months), contemplation (considering behaviour change between one and six months), preparation (getting ready to change behaviour in the next month), action (performing the change in behaviour), and maintenance (steady stage of behaviour change reached). In the feedback, individuals receive a health plan that fits with their current stage of change. Participants in earlier stages receive information to motivate them to change their lifestyle and participants in later stages receive concrete action plans how to change their lifestyle behaviour.

\section{Tailored feedback}

Participants receive tailored feedback based on their responses to the HRA questionnaire and additional measurements if indicated. The feedback contains a health risk profile divided into four domains: lifestyle, psychological, social (including work-related aspects) and physical, and each of these divided into sub-domains (Table 1). For each of these domains the assessed health risk is explained using a simple grid labelled by one of three colours: green representing 'normal risk', orange representing 'moderately elevated risk' and red representing 'seriously elevated risk'. In addition, threats associated with elevated risk (orange and red categories) and potential gains of taking preventive action are explained. The feedback concludes with an overview of actions the participant can take. The number of possible actions depends on the outcome of the HRA. All options include suggestions from trusted external parties, where participants could go for support for the action they want to take. The suggestions are made to fit with their expressed preferences, such as for guided vs. nonguided interventions.

In summary, differentiations are made between the stage of motivation (see 'Theoretical framework for lifestyle change'), the preferences for professional guidance (yes/ no), actions in groups or alone, actions outside or close to home, and actions via internet, telephone or face-to-face. In case of high risk (red category), the feedback includes referral for further medical evaluation and treatment if necessary.

\section{Evaluation study \\ Study population and procedure}

All employees aged $\geq 18$ years from companies participating in the study are eligible for participation in the evaluation study. Individual participation in the study is on a voluntary basis. Employees in the intervention group receive an invitation for participation in the HRA at baseline. Their completion of the lifestyle items in the questionnaire of the HRA serves two purposes: it belongs to their HRA but also acts as a baseline measurement of health behaviour for the evaluation study. Six months later participants receive an invitation for a short, electronic lifestyle questionnaire that serves as follow-up measurement.

Employees in the control group receive an invitation for the short, electronic lifestyle questionnaire at baseline. In this group this questionnaire serves as the baseline measurement. Six months later control group participants receive an invitation to take part in the HRA. The completion of the lifestyle items in the questionnaire of the HRA now serves as follow-up measurement (Figure 1).

Allocation to the intervention or control group Individual allocation may lead to contamination between the groups in the case that collaborating employees are allocated to different groups; this could then imply that the results are influenced by factors other than the intervention. Therefore, we rely on allocation by cluster. Within each company, clusters of employees with comparable activities and of comparable size are allocated to either the intervention or the control group.

Due to practical limitations, random allocation of clusters to the intervention and control group is not possible. Both the intervention and the study have to be embedded in the corporate health management strategy of the participating companies. Allocation of units to the intervention and control group will follow the roll-out procedure of the company, i.e. by geographical region and mainly based on practical considerations of the company management like current reorganization. Although the allocation procedure 
Table 1 Health-related domains and sub-domains on which participants of the health risk assessment receive tailored feedback

\begin{tabular}{llll}
\hline Lifestyle domain & Psychological domain & Social domain & Physical domain \\
\hline Physical activity & Stress & Workability/productivity & Blood-pressure* $^{*}$ \\
\hline Smoking behaviour & Burn-out & Work satisfaction & Lipids* $^{*}$ \\
\hline Alcohol consumption & Depression & Work-home balance & Blood sugar* \\
\hline Nutrition & Anxiety & Interaction with colleagues & Kidney function* \\
\hline & & & Body weight and fat distribution \\
\hline
\end{tabular}

*Only if additional biometric and laboratory evaluation are performed.

is not random, confounding is unlikely; conversations with the management teams of companies confirm that clusters could not obtain priority in the allocation procedure.

Furthermore, effects of this limitation on the estimate of the effect of the intervention will be minimized by adjusting for baseline characteristics of clusters in the statistical analysis (see 'Statistical analysis').

Invitation for the lifestyle questionnaire (control group) Employees are invited through a letter to their home address to complete the lifestyle questionnaire via Internet. An information leaflet about the evaluation study is enclosed. The invitation letter informs employees that participation is voluntary, that all personal data are treated confidentially, and that no result is shared with their employer and only used in anonymous form for the present study. The invitation letter contains a unique number for access to the questionnaire via the website. In case of no response to the letter of invitation, participants receive a single reminder after three weeks, in the form of a letter to their home address.

\section{Measurements}

The responses to the HRA questionnaire are used for two purposes. First, the responses form the basis for generation of the tailored feedback. Second, the responses to the lifestyle items are used to analyse the outcome measures of this evaluation study.

Primary outcome is lifestyle behaviour, expressed as the sum of five indicators (physical activity, nutrition, smoking behaviour, alcohol consumption and symptoms of burnout). For each indicator, adherence to the Dutch guidelines (yes/no) is determined. The magnitude of change in lifestyle behaviour measured after six months determines whether or not the intervention is successful.

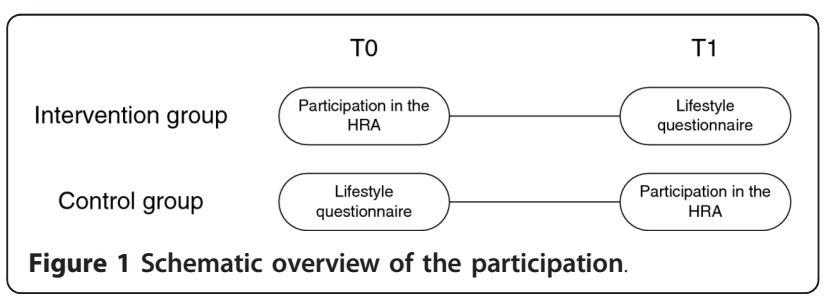

Physical activity Physical activity is measured with two items derived from the Dutch version of the International Physical Activity Questionnaire (IPAQ) [16]. These two closed-ended items provide information on the frequency (days per week) and duration (minutes) spent on moderate intensity and vigorous physical activity in a normal week.

The public health recommendation for physical activity in the Netherlands, the Dutch Healthy Physical Activity Guideline (Nederlandse Norm Gezond Bewegen), recommends physical activity for $30 \mathrm{~min}$ (or more) per day at a moderate or vigorous intensity level, for at least 5 days a week [3]. Adherence to this guideline is measured with one additional item about the number of days that the participant is physically active for $30 \mathrm{~min}$ (or more) per day at a moderate or vigorous intensity level.

To generate the feedback, the total duration of physical activity in min/week is calculated, collating moderate and vigorous activities. Additionally, total physical activity duration is dichotomized into $\leq 150 \mathrm{~min} /$ week or $\geq 150$ $\mathrm{min} /$ week, as a second way of reflecting adherence to the Dutch Healthy Physical Activity Guideline.

For the evaluation study, adherence to the cardiorespiratory fitness guideline (Fitnorm) is also analyzed. This guideline recommends physical activity for $20 \mathrm{~min}$ (or more)/day at a vigorous intensity level, for at least 3 days/week [2]. Adherence to this norm is analyzed using the questions described above. Total physical activity duration is dichotomized into $\leq 60 \mathrm{~min} /$ week or $\geq 60 \mathrm{~min} /$ week. The cardio-respiratory fitness guideline is not used in the tailored feedback of the intervention.

Nutrition Different aspects of the dietary pattern are taken into account, as defined by the Dutch Dieticians Cooperation: fruit and vegetables, saturated fat intake, intake of sugar, and fish.

Items concerning fruit and vegetables are based on a standard nutrition questionnaire of the Dutch Municipal Health Service [17]. Participants are asked to report their fruit intake in pieces of fruit per day in a normal week. They also are asked to report the number of serving spoons of vegetables per day in a normal week. In the Netherlands, a daily intake of at least 2 pieces of fruit and 
$200 \mathrm{~g}$ of vegetables (4 spoons per day, defining a serving spoon of vegetables as $50 \mathrm{~g}$ ) is the public health recommendation [18].

Saturated fat intake is measured with items developed in expert meetings. Participants are asked to report the amount of snacks, use of butter, cheese and meat (portions/day), dairy products and sweets. To generate the feedback each answer has a score that cumulatively leads to a total score for saturated fat intake. Experts defined a cut-off value that is used to determine the colour (green/ orange) used in the feedback.

The intake of sugar is based on the amount of teaspoons of sugar, as well as sweet drinks and sweet snacks consumed per day. Again, to generate the feedback, each answer has a score that cumulatively leads to a total score for intake of sugar; a daily intake of $40 \mathrm{~g}$ or less is recommended.

Also noted are the frequency of breakfast (days/week) and the frequency of fish (portions/week). The public health recommendation of frequency of breakfast is every day, thus 7 days a week [18]. For fish, this recommendation includes fish consumption at least two times a week, of which at least one portion fatty fish [18]. All aspects of the dietary pattern are dichotomized according to the guidelines, to generate the feedback.

Smoking behaviour Smoking behaviour is measured by items from a questionnaire of the Dutch Expert Centre on Tobacco Control (STIVORO) [19]. To generate the feedback, participants are categorized into non-smokers, ex-smokers, incidental smokers, daily smokers and heavy smokers. A heavy smoker is defined as smoking 10 or more units of tobacco per day. The evaluation study only incorporates the questions if the participant is currently smoking (yes/no), and then the kind of tobacco, and the units of tobacco per day.

Alcohol consumption Alcohol consumption is measured in units of alcohol per day based on a standard alcohol questionnaire of the Dutch Municipal Health Service [20]. For men and woman different limits for excessive alcohol consumption are used; for men alcohol consumption up to 21 units/week and for women alcohol consumption up to 14 units/week is recommended as maximum. Furthermore, alcohol dependence is measured using the 'fiveshot' questionnaire [21]. This questionnaire gives an initial indication concerning alcohol dependence via 5 questions. Each answer has a score that contributes to a total score of alcohol dependence. The total score has a defined cutoff value for alcohol dependence. To generate the feedback, the units of alcohol per day and the score of the five-shot questionnaire are dichotomized according to the norm.

Burnout The UBOS questionnaire is used for symptoms of burnout [22]; this questionnaire is the Dutch version of the the Maslach Burnout Inventory [23]. Each answer has a score that contributes to a total score of indication for burnout. The total score has a defined cut-off value. To generate the feedback, indication for burnout is dichotomized according to this value.

\section{Determinants of behavioural change}

Additional determinants of health behavioural change are measured, as described below.

Intention to behaviour change The intention to change behaviour is measured per lifestyle variable. Answer options are based on the different phases of Prochaska's 'Stage of Change' model [15], and are formulated as: 'I don't have any intention to change my lifestyle between one and six months', 'I am considering to change my lifestyle within between one and six months', 'I'm getting ready to change my lifestyle in the next months', 'I'm already working to improve my lifestyle', and 'I have reached the intended lifestyle behaviour and still perform it'.

Self-efficacy Self-efficacy is measured by one newly developed item where participants have to indicate to what extent they feel able or unable to change their lifestyle behaviour if necessary. This item is formulated as: 'I am able to change my lifestyle behaviour if necessary'. Answers are rated on a 5-point Likert scale ranging from 1 (totally agree) to 5 (totally disagree).

Social support Social support is measured by three items; two reflecting the support for participating in a HRA with tailored feedback from the respondent's work environment (colleagues and managers), and one reflecting the support from the respondent's partner and/or family. Respondents are asked to complete the statements: 'According to my colleagues, participation in a HRA with tailored feedback is useful', 'According to my manager, participation in a HRA with tailored feedback is useful', and 'According to my partner and/or family, participation in a HRA with tailored feedback is useful'. Answers are rated on a 5-point Likert scale ranging from 1 (very useful) to 5 (totally useless) and an additional answer option 'I don't know'.

\section{Confounding variables}

Non-random allocation to the intervention and control group may result in an unequal distribution of the characteristics of employees and/or companies and their units, which can influence the changes in lifestyle behaviour. Measured potential confounders of the change in lifestyle behaviour include age, gender and educational level, years of work at the current company, years in current function, and part-time or fulltime employment.

\section{Statistical analysis}

Descriptive statistics will be used to describe and compare baseline characteristics of participants and clusters in the intervention and control group. Any baseline differences with a potential effect on lifestyle change will be taken into account in further analyses using propensity score weighting. To estimate the effect of the intervention on 
lifestyle behavioural change logistic regression analysis (for binary outcome measures) and linear regression analysis (for continuous outcome measures) will be used. To correct for a potential clustering effect, multilevel analyses will be performed. In case the follow-up measurement result in a considerable amount of missing data, multiple imputation methods will be used.

\section{Power analysis}

The sample size calculation is based on the primary outcome, lifestyle behaviour, expressed as the sum of five indicators. We consider a difference in a range of 0.3 to 0.5 points more behavioural change in the intervention group compared to the control group as a meaningful effect (power of 80\%; one-sided significance level, 0.05). With an intra-cluster correlation of 0.05 (as in a previous study among employees [24]), and an average of 30 employees per cluster (based on 100 eligible employees per cluster, 30\% initial participation), we must include at least 17 clusters per group, thus a total of 1020 employees, to find a difference of 0.3 points. To find a difference of 0.5 point we have to include at least 7 clusters per group, thus a total of 420 employees.

\section{Discussion}

The study presented here is designed to evaluate the effectiveness of a health risk assessment with tailored feedback conducted in a worksite setting, on lifestyle behaviour. The rationale for this study is the wide availability and use of worksite HRAs, in combination with the lack of evidence concerning their impact on health behaviour.

Results may be relevant for future development and implementation of web-based interventions aimed at improving lifestyle behaviour relevant for prevention of cardiovascular diseases and cancer. In the present study, the broad approach of the evaluated HRA, and the fact that feedback is tailored to participants' characteristics, make it of potential interest for all individuals and not only for those at high risk. It creates awareness of unhealthy behaviour before chronic disease symptoms are present, by providing information about (un)healthy behaviour in the health recommendations. This is a crucial step for those not yet ready for behavioural change.

The fact that the present evaluation is performed in a real-life setting is a strength, because this provides a realistic estimate of effectiveness. The controlled design allows for evaluation of the effect, in addition to autonomic change in lifestyle behaviour.

Potential sources of bias include the Hawthorne effect, registration bias, and carry-over bias. The Hawthorne effect can occur in individuals who change their behaviour due to the attention they receive from, e.g. the researchers. In this case, however, the effect is probably limited because the intervention is mostly provided online with little contact with the researchers. Bias due to participants giving socially desirable answers is a possibility when using self-report questionnaires; although we aim to minimise such bias by formulating the items in a neutral way and using validated questionnaires where possible, it cannot be excluded. Finally, we aim to limit carry-over bias by the use of cluster randomization.

Taking into account the benefits of a worksite setting and the web-based delivery of the HRA, we believe this HRA is applicable to and attractive for the population at large. This study has the potential to make a substantial contribution to the development of effective interventions for lifestyle behaviour change among a working population. Results are expected to be available in 2013.

\section{Acknowledgements}

This study is funded by ZonMw, the Netherlands Organisation for Health Research and Development. ZonMw project number 200310006.

\section{Author details}

${ }^{1}$ Department of Public Health, Academic Medical Center, Amsterdam, The Netherlands. ${ }^{2}$ NDDO Institute for Prevention and Early Diagnostics (NIPED), Amsterdam, The Netherlands. ${ }^{3}$ Department of Medical Informatics, Academic Medical Center, Amsterdam, The Netherlands. ${ }^{4}$ Department of Clinical Epidemiology, Biostatistics and Bioinformatics (KEBB), Academic Medical Center, Amsterdam, The Netherlands.

\section{Authors' contributions}

MD designed and wrote the original proposal, supported by KS, PB and RK. EL further developed the study protocol and is responsible for data collection, data analysis, and drafting of the final research report, supervised by MLE, NP and RK. All authors participated in discussing the design of the study and developing the research protocol. All authors have read and approved the final manuscript.

\section{Competing interests}

Roderik A Kraaijenhagen is medical director and co-owner of NIPED. This institute developed and currently markets the studied program in the Netherlands. NIPED assists several research programs for scientific quality assurance purposes that are conducted by academic institutions. The present study is an example of such a project. During this research, Eva K Laan was employed at NIPED on a part-time basis ( $4 \mathrm{~h} /$ week) for which she received a salary.

Received: 16 February 2012 Accepted: 19 March 2012 Published: 19 March 2012

\section{References}

1. World Health Organization: Burden: mortality, morbidity and risk factors. In Global status report on noncommunicable diseases 2010 Geneva; 2011.

2. In Trendrapport Bewegen en Gezondheid 2006/2007 [Trend Report Physical Activity and Health 2006/2007]. Edited by: Hildebrandt VH, Ooijendijk WTM, Hopman-Rock M. Leiden, Netherlands, TNO; 2008:

3. Kemper HCG, Ooijendijk M, Stiggelbout M: Consensus over de Nederlandse norm voor gezond bewegen [Consensus about the Dutch guideline for healthy physical activity]. Tijdschrift voor gezondheidswetenschappen 2000, 78:180-183.

4. Hulshof KFAM, Ocké MC, van Rossem CTM, Buurma-Rethans EJM, Brants HAM, Drijvers JJMM, ter Doest D: Resultaten van de Voedselconsumptiepeiling 2003 [Results of the national food consumption survey 2003] (in Dutch RIVM Rapport 350030002) Netherlands, RIVM: Bilthoven; 2004.

5. In Volksgezondheid toekomst verkenning; deelrapport Gezondheid en determinanten [Public Health Forecast; Health and determinants.] (in Dutch RIVM Rapport 270061006). Edited by: Schoemaker CG, Hoeymans N, Melse JM. Bilthoven, Netherlands, RIVM; 2010: 
6. Dishman RK, Oldenburg B, O'Neal H, Shephard RJ: Worksite physical activity interventions. Am J Prev Med 1998, 15(Suppl 4):344-361.

7. Goetzel RZ, Pronk NP: Worksite health promotion: how much do we really know about what works? Am J Prev Med 2010, 38(Suppl 2):223-225.

8. Pelletier KR: A review and analysis of the clinical and cost-effectiveness studies of comprehensive health promotion and disease management programs at the worksite: update VIII 2008 to 2010. J Occup Environ Med 2011, 53(Suppl 11):1310-1331.

9. Soler RE, Leeks KD, Razi S, Hopkins DP, Griffith M, Aten A, et al: A systematic review of selected interventions for worksite health promotion. The assessment of health risks with feedback. Am J Prev Med 2010, 38(Suppl 2):237-262.

10. Brug J, Oenema A, Kroeze W, Raat H: The Internet and nutrition education: challenges and opportunities. Eur J Clin Nutr 2005, 59:130-139.

11. Franklin PD, Rosenbaum PF, Carey MP, Roizen MF: Using sequential email messages to promote health behaviors: evidence of feasibility and reach in a worksite sample. J Med Internet Res 2006, 8(Suppl 1):e3.

12. Riper H, Kramer J, Smit F, Conijn B, schipper G, Cuijpers P: Web-based selfhelp for problem drinkers: a pragmatic randomized trial. Addiction 2008, 103:218-227.

13. Burgers JS, Simoons ML, Hoes AW, Stehouwer CD, Stalman WA: Richtlijn cardiovasculair risico management [guideline cardiovascular risk management]. Ned Tijdschr Geneeskd 2007, 151(19):1068-1074.

14. Graham I, Atar D, Borch-Johnsen K, Boysen G, Burell G, Cifkova R, Dallongeville J, de Backer G, Ebrahim S, Gjelsvik B, Herrmann-Lingen C, Hoes A, Humphries S, Knapton M, Perk J, Priori SG, Pyorala K, Reiner Z, Ruilope L, Sans-Menendez S, ScholteopReimer W, Weissberg P, Wood D, Yarnell J, Zamorano JL: European guidelines on cardiovascular disease prevention in clinical practice: executive summary. Eur Heart J 2007, 28(19):2375-2414.

15. Prochaska JO, Velicer WF: The transtheoretical model of health behaviour change. Am JHealth Promot 1997, 12(Suppl 1):38-48.

16. Craig CL, Marshall AL, Sjostrom M, Bauman AE, Booth ML, Ainsworth BE, Pratt M, Ekelund U, Yngve A, Sallis JF, Oja P: International physical activity questionnaire: 12-country reliability and validity. Med Sci Sports Exerc 2003, 35:1381-1395.

17. Brink CLvd, Ocké MC, Houben AW, Nierop Pv, Droomers M: Validering van standaardvraagstelling voeding voor Lokale en Nationale Monitor Volksgezondheid IValidation of a Community Health Services food consumption questionnaire in the Netherlands] (in Dutch RIVM Rapport 260854008) Bilthoven: RIVM; 2005

18. Health Council of the Netherlands: Richtlijnen goede voeding 2006 [Guidelines for a healthy diet 2006], [Richtlijnen goede voeding 2006] Den Haag; 2006.

19. In Meetinstrumenten voor onderzoek naar roken en stoppen met roken [Measurement instrument for research on smoking and quitting smoking]. Edited by: Mudde AN, Willemsen MC, Kremers S, Vries Hd. Den Haag, Netherlands, Stivoro; 2006:

20. Lokale en nationale monitor gezondheid [Health Monitor in the Netherlands]. [https://www.monitorgezondheid.nl/volksindicatoren.aspx]

21. De RA, Vanheule S: Pilot study: does the five shot questionnaire give an indication of the severity of alcohol use-related problems? Subst Use Misuse 2007, 42:1593-1601.

22. Schaufeli WB, van Dierendonck D: Utrechtste Burnout Schaal (UBOS) [Utrechtse Burnout Scale (UBOS)]. De psycholoog 2001, 36:9-12.

23. Schaufeli WB, Bakker AB, Hoogduin K, Schaap C, Kladler A: On the clinical validity of the Maslach Burnout Inventory and the burnout measure. Psychol Health 2001, 16:565-582.

24. ljzelenberg $H$, Meerding WJ, Burdorf A: Effectiveness of a back pain prevention program: a cluster randomized controlled trial in an occupational setting. Spine 2007, 32(Suppl 7):711-719.

\section{Pre-publication history}

The pre-publication history for this paper can be accessed here: http://www.biomedcentral.com/1471-2458/12/200/prepub

doi:10.1186/1471-2458-12-200

Cite this article as: Laan et al: Effectiveness of a web-based health risk assessment with individually-tailored feedback on lifestyle behaviour: study protocol. BMC Public Health 2012 12:200.

\section{Submit your next manuscript to BioMed Central and take full advantage of:}

- Convenient online submission

- Thorough peer review

- No space constraints or color figure charges

- Immediate publication on acceptance

- Inclusion in PubMed, CAS, Scopus and Google Scholar

- Research which is freely available for redistribution

Submit your manuscript at www.biomedcentral.com/submit 